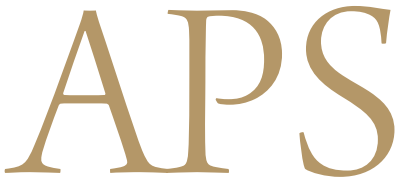

Archives of Plastic Surgery

\title{
The Clinical Usefulness of Ultrasound-Aided Fixation Using an Absorbable Plate System in Patients with Zygomatico-Maxillary Fracture
}

\author{
Jong Hun Lee ${ }^{1,2}$, Jun Hyung Park ${ }^{1}$ \\ ${ }^{1}$ Department of Plastic and Reconstructive Surgery, ${ }^{2}$ Eulji Medi-Bio Research Institute, Eulji General Hospital, Eulji University School of \\ Medicine, Seoul, Korea
}

Background Ultrasound-aided fixation is a recently developed alternative method of treatment of zygomatico-maxillary (ZM) fracture, and it can resolve the problems of excessive torsion force and subsequent fractures of screws. We conducted this study to evaluate the clinical usefulness of ultrasound-aided fixation as compared with the conventional fixation method using a drill and an expander in patients with ZM fracture.

Methods We conducted a retrospective study in 35 patients with ZM fracture who had been treated at our hospital during a period ranging from March of 2008 to December of 2010. We divided them into two groups: an ultrasound-aided fixation group, comprising 13 patients who underwent ultrasound-aided fixation (SonicWeld Rx, KLS Martin), and a conventional group, comprising 22 patients who underwent conventional fixation (Biosorb FX, Linvatec Biomaterials Ltd.). We compared such variables as sex, direction, age at operation, follow-up period, operation duration, number of fixed holes, and time to discharge between the two groups.

Results The ultrasound-aided fixation reduced the operation duration by about 30 minutes as compared with that of conventional fixation. There was no significant difference in followup period, number of fixed holes, or time to discharge between the two groups. Furthermore, there were no complications in either group.

Conclusions The ultrasound-aided fixation of fractured ZM bone using an absorbable implant system is safe and effective in promptly reducing the bone fracture and providing satisfactory cosmetic outcomes over time.

Keywords Facial bone / Fractures, bone / Ultrasonics / Bone plates

\author{
Correspondence: Jong Hun Lee \\ Department of Plastic and \\ Reconstructive Surgery, Eulji General \\ Hospital, Eulji University School of \\ Medicine, 14 Hangeulbiseok-gil, \\ Nowon-gu, Seoul 139-711, Korea \\ Tel: +82-2-970-8255 \\ Fax: +82-2-978-4772 \\ E-mail: joaljh@eulji.ac.kr
}

This article was presented at the 69th Congress of the Korean Society of Plastic and Reconstructive Surgeons on November 11-13, 2011 in Seoul, Korea.

No potential conflict of interest relevant to this article was reported.

Received: 21 Dec 2012 • Revised: 28 Jan $2013 \bullet$ Accepted: 24 Apr 2013

pISSN: 2234-6163 • elSSN: 2234-6171 • http://dx.doi.org/10.5999/aps.2013.40.4.330 • Arch Plast Surg 2013;40:330-334

\section{INTRODUCTION}

In the conventional absorbable plate system, rigid fixation is achieved using screw- or tack-based methods. Absorbable screws require bone drilling and tapping prior to screw placement. In addition, poor screw purchase can occur if the proper technique is not used. Tack fixation requires that a significant amount of force be applied to the tack to drive it securely into the bone. However, there are reported disadvantages such as prolonged operation time and complicated surgical handling because of

Copyright (๑) 2013 The Korean Society of Plastic and Reconstructive Surgeons

This is an Open Access article distributed under the terms of the Creative Commons Attribution Non-Commercial License (http://creativecommons.org/

licenses/by-nc/3.0/) which permits unrestricted non-commercial use, distribution, and reproduction in any medium, provided the original work is properly cited.

www.e-aps.org 
the need to tap threads into the screw holes $[1,2]$.

Ultrasound-aided fixation is an alternative method that has been recently developed. The absorbable pin is mounted at the tip of a device called a sonotrode, which then vibrates the pin to ultrasonic speeds. As the vibrating pin is inserted into the hole drilled into the bone, the friction generated at the pin-bone interface causes the pin edges to melt into the bone, resulting in a flow of biodegradable polymers into the cortical bone layer and the cavities of the cancellous bone. This can resolve the problems of excessive torsion force and subsequent fractures of screws. Theoretically, the fusion of the plate and pinhead is the definitive characteristic of a mechanical device superior to conventional fixation. Besides, the advantages of ultrasound-aided fixation include optimum operative handling, reduced insertion time, avoidance of fractures of the fixation elements, and higher three-dimensional load capacity [1].

Given the above background, we conducted this study to evaluate the clinical usefulness of ultrasound-aided fixation as compared with the conventional fixation method based on a drill and an expander in patients with zygomatico-maxillary (ZM) fracture.

\section{METHODS}

\section{Study patients}

We conducted the current retrospective study in 35 patients with ZM fracture who had been treated at our hospital during a period ranging from March of 2008 to December of 2010. All of our patients were operated on by a single surgeon. We divided them into two groups: the ultrasound-aided fixation group, comprising 13 patients who underwent ultrasound-aided fixation (SonicWeld Rx, KLS Martin, Tuttlingen, Germany), and the conventional fixation group, comprising 22 patients who underwent conventional fixation (Biosorb FX, Linvatec Biomaterials Ltd., Tampere, Finland). We compared such variables as sex, direction, age at operation, follow-up period, operation time, number of fixed holes, and time to discharge between the two groups. The operation time was measured as the total operating time except for the Gilles' operation time. The date of the last follow-up was determined by that of the last outpatient visit. Postoperatively, all of the patients were followed up for clinical and radiological findings.

\section{Surgical technique}

Surgical treatment was performed within 3 to 7 days after the onset of trauma, depending on the amount of swelling, through an intraoral and a subciliary incision. In addition, we performed Gilles' operation for the reduction of zygomatic arch fracture.
An intraoral incision was made in the buccal vestibule from the canine to the first molar area. The fractured segment of the $\mathrm{ZM}$ bone was exposed up to the inferior orbital margin using a subperiosteal dissection. This was followed by the exposure of the orbicularis oris muscle through a subciliary incision. Then a blunt dissection was performed in the direction of the muscle fibers. Moreover, an incision was also made in the periorbital periosteum, followed by the exposure of the infraorbital rim and the upper part of the fractured area using a subperiosteal dissection. Repositioning of the bony fragments was conducted. Then, 3 absorbable plates with 4 or 6 holes were properly located above the fractured site.

In the conventional fixation group, a hole was made in the fracture fragment using a drill. This was followed by the insertion of the tapper in the hole. Then, the plate and the fracture fragment were fixed tightly by absorbable screws. In addition, if needed, extra screws were inserted in other sites. In the ultrasound-aided fixation group, various types of plates were available for fixation. These plates could be molded and then shaped in hot water. The sonotrode was attached to a base unit that generated the ultrasonic frequency. The absorbable pin was mounted to the tip of the sonotrode and then inserted in the hole made in the fractured segment of the ZM bone through the plate. In the ultrasound-aided fixation group, the absorbable pin was set to vibrate with the tip of the sonotrode. The pin was then placed into the bone with minimal pressure and absorbed into the drilled hole. At the completion of the pin insertion, the application of the ultrasonic frequency was discontinued. Then, gentle pressure was applied to the pin and sonotrode for 1 to 2 seconds before the sonotrode was extracted. Thus, the pin was fixed in the hole (Fig. 1). After hemostasis was achieved, a drainage tube was inserted in the surgical sites. Following this, the wound was closed layer by layer.

\section{Statistical analysis}

Statistical analysis was done using SPSS ver. 14.0 (SPSS Inc., Chicago, IL, USA). All of the the data was expressed as mean \pm standard deviation. We used Fisher's exact test to compare such variables as the sex and the location of the fracture. In addition, we performed an independent samples t-test to compare such variables as the mean age at operation, the mean follow-up period, the operation time, and the time to discharge between the two groups. A P-value of $<0.05$ was considered statistically significant.

\section{RESULTS}

As shown in Table 1, there were no significant differences in baseline characteristics such as the mean age and the mean follow-up 


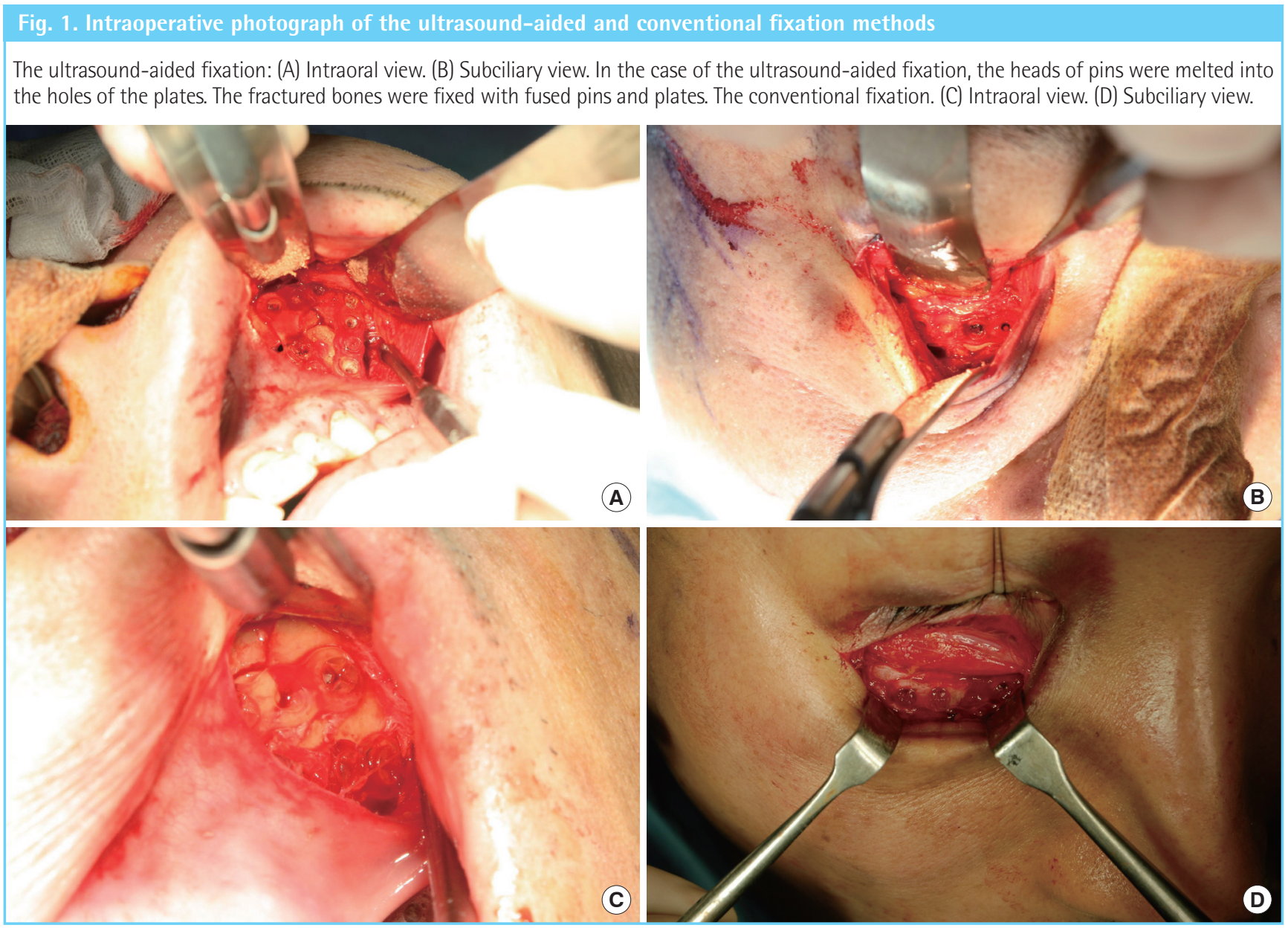

period between the two groups. In the conventional fixation group $(n=22)$, comprising 21 men and one woman, the mean age at operation was 32.6 years (range, 11 to 65 years) and the mean follow-up period was 210.4 days (range, 60 to 536 days). In addition, there were 6 right-sided cases and 16 left-sided cases. Furthermore, there were no bilateral cases. In the ultrasound-aided fixation group $(\mathrm{n}=13)$, comprising 13 men and no women, the mean age at operation was 31.2 years (range, 15 to 55 years) and the mean follow-up period was 190.9 days (range, 96 to 248 days). In addition, there were 5 right-sided cases and 8 left-sided cases. Furthermore, there were no bilateral cases.

Additionally, there was a significant difference in the operation time between the two groups. That is, it took significantly shorter to reconstruct the ZM fracture in the ultrasound-aided fixation group as compared with the conventional fixation group (90 minutes vs. 119.9 minutes, $P=0.0269$ ). This showed that the ultrasonic-aided fixation was more advantageous than the conventional one. For the ultrasound-aided fixation, an average of 9.9 pins and 10.1 screws were used. There was no significant difference in the number of pins or screws used between the two groups $(\mathrm{P}=0.6446)$. Therefore, the operation time of the

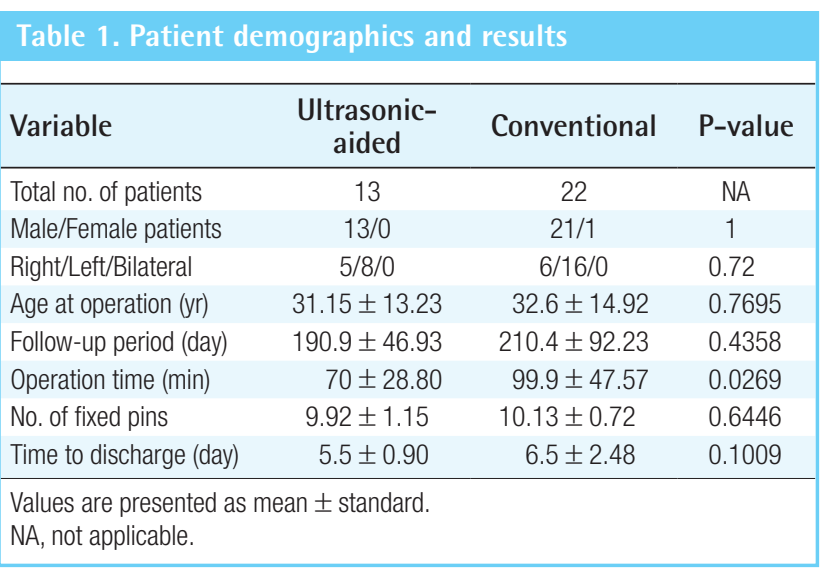

ultrasound-aided fixation group was shorter by about 3 minutes per hole than that of the conventional fixation group. In addition, there was no significant difference in the time to discharge between the two groups ( 5.5 days vs. 6.5 days, $\mathrm{P}=0.1009$ ) (Table 1). Furthermore, there were no complications in either group. Furthermore, the patients were cosmetically satisfied with the postoperative appearance. 


\section{DISCUSSION}

Various types of hardware such as plates and screws are used to reduce facial bone fractures and have replaced wire fixation, thus providing instantaneous rigid fixation of the bone. This remains a popular method of fixation, particularly in cases in which there is a need for immediate structural support of the bone fragments. The metal plate and screw provide rigid fixation and stability. However, they also carry the risk of migration with growth, skin erosion, aseptic necrosis, subjective discomfort, infection, bone growth restriction, and the radiation scatter effect [1,3-5].

Fixation using an absorbable plate system was developed in the 1990s, and it has gained wide use in the field of pediatric cranial and facial surgery [6]. Such systems have the advantages of immediate rigid fixation without the use of long-term implants. The biodegradable plates and screws have several advantages over titanium plates and screws, and these include 1) no need for a secondary intervention to remove the devices, 2) no interference with imaging or radiotherapeutic techniques, 3) no possible growth disturbance or mutagenic effects, and 4) no thermal sensitivity $[7,8]$. Moreover, there is no cellular reaction due to thermal stress during insertion [1] Furthermore, there are rare occurrences of device-related complications including device failure secondary to the external trauma, delayed foreign body reactions, and granuloma formation $[5,6]$.

One of the limitations of biodegradable implant systems is the method of fixation of the plates or mesh to the bone. The screwbased fixation requires tapping of the screw threads before insertion because the screws are not self-tapping [5]. This step can be quite burdensome and can result in hand fatigue, particularly if multiple screws are involved [6]. Furthermore, the screw heads can fracture from the shaft if the length of the threaded screw path is inadequate or if the longitudinal axis of the screw is not aligned with the threaded screw path in an orthograde fashion [1]. The hand tapping of the thread can be time-consuming. In addition, downward pressure is required when it is done with screw placement. This can increase the difficulty of placement [5].

Our results showed that ultrasound-aided fixation using rigid absorbable implants in facial surgery provided rigid bone fixation with no complications. These rigid absorbable implants are composed of a 50:50 mixture of D-isomers and L-isomers of polylactic acid $[4,5]$. According to the manufacturer's instructions, the loss of the implants' initial strength appears 8 to 10 weeks after the tissue implantation. In addition, complete resorption of the implant will occur by approximately two years later. The D-isomers and L-isomers of polylactic acid are hydrolyzed through phagocytic internalization by macrophages [5].
This is a signal transduction pathway by which fibroblast-like cells are attracted and the final products such as $\mathrm{H}_{2} \mathrm{O}$ and $\mathrm{CO}_{2}$ are released [9]. The polymers are fabricated into various plates, meshes, or buttons, and can be fixed to the skull or facial bones using screws, tacks, or pins $[2,10]$. Furthermore, the ultrasoundaided fixation system was advantageous in that the pin could be implanted in a two-stage procedure. That is, it is advantageous in that there is no need to tap a screw thread or turn the screw and this makes it possible to promptly implant the pin. Our results showed that the operation time of the ultrasound-aided fixation group was shorter by about 3 minutes per hole as compared with duration for the conventional fixation group. However, there is a possibility that the operation time gap could be elongated, such as when a screw is fractured during fixation, or tapping one is insufficient, or a dropped screw has to be retried due to an improperly locking screw.

Moreover, the ultrasound-aided fixation system is useful for thin bone because it can disperse pressure and thereby provide a rivet-like retention. In addition, minimal forces are required for the insertion of the pin. This indicates that it is less probable that the fracture of the thin bone might occur. It can also be inferred that the pin can be safely placed over vital structures such as vessels and nerves. Therefore, the ultrasound-aided fixation system would be an ideal modality for thin cortical bone, cancellous (porous) bone, and the areas that are adjacent to vital structures. There are some limitations of the current study, whose results cannot be generalized to the fractures of other facial regions. To rule out the factors that vary among sites and thus differentiate between the two fixation systems, only ZM fractures were considered in this study. Further studies are, therefore, warranted to apply our results to the fractures of other facial regions. In addition, based on the claims of the manufacturer, the complete absorption of the plate would not be achieved for approximately two years, leaving a window of time in which foreign body reactions such as granuloma formation could occur. In the current study, however, the mean follow-up period was eight months. Further long-term follow-up studies are warranted to support our findings. Computed tomography (CT) is effective in the postoperative assessment of bone fracture malposition and malunion. However, postoperative CT was not performed in all patients in our study. Further follow-up CT studies are warranted to support our results.

Our results showed that the ultrasound-aided fixation of fractured ZM bone using an absorbable implant system is a safe, effective method for promptly reducing the bone fracture and providing a satisfactory cosmetic outcome over time. 


\section{REFERENCES}

1. Pilling E, Meissner H, Jung R, et al. An experimental study of the biomechanical stability of ultrasound-activated pinned (SonicWeld Rx+Resorb-X) and screwed fixed (Resorb-X) resorbable materials for osteosynthesis in the treatment of simulated craniosynostosis in sheep. Br J Oral Maxillofac Surg 2007;45:451-6.

2. Suuronen R, Kallela I, Lindqvist C. Bioabsorbable plates and screws: current state of the art in facial fracture repair. J Craniomaxillofac Trauma 2000;6:19-27.

3. Berryhill WE, Rimell FL, Ness J, et al. Fate of rigid fixation in pediatric craniofacial surgery. Otolaryngol Head Neck Surg 1999; 121:269-73.

4. Cho PW, Burton RG, Gratton D, et al. Biomechanical study of SonicWeld $\mathrm{Rx}$ pin in cortical bone graft layering technique. J Oral Maxillofac Surg 2011;69:1519-24.

5. Aldana PR, Roy S, Postlethwait RA, et al. Ultrasound-aided fixation of a biodegradable cranial fixation system: uses in pediatric neurosurgery. J Neurosurg Pediatr 2009;3:420-4.

6. Eppley BL, Morales L, Wood R, et al. Resorbable PLLAPGA plate and screw fixation in pediatric craniofacial surgery: clinical experience in 1883 patients. Plast Reconstr Surg 2004; 114:850-6.

7. Buijs GJ, van der Houwen EB, Stegenga B, et al. Mechanical strength and stiffness of the biodegradable SonicWeld $\mathrm{Rx}$ osteofixation system. J Oral Maxillofac Surg 2009;67:782-7.

8. Enislidis G, Pichorner S, Lambert F, et al. Fixation of zygomatic fractures with a new biodegradable copolymer osteosynthesis system. Preliminary results. Int J Oral Maxillofac Surg 1998;27:352-5.

9. Bergsma JE, de Bruijn WC, Rozema FR, et al. Late degradation tissue response to poly (L-lactide) bone plates and screws. Biomaterials 1995;16:25-31.

10. Suuronen R. Biodegradable fracture-fixation devices in maxillofacial surgery. Int J Oral Maxillofac Surg 1993;22:50-7. 